ONLINE MEDICAL GENETICS IN PRACTICE

\title{
Bayesian risk assessment for autosomal recessive diseases: fetal echogenic bowel with one or no detectable CFTR mutation
}

\author{
S Ogino, R B Wilson, W W Grody
}

J Med Genet 2004;41:e70 (http://www.jmedgenet.com/cgi/content/full/41/5/e70). doi: 10.1136/jmg.2003.015065

$\mathrm{R}$ isk assessment is an essential component of genetic counselling and testing, and Bayesian analysis plays a central role in complex risk calculations. ${ }^{1-3}$ Prenatal risk assessment for autosomal recessive diseases can be particularly complex when, for example, only one mutation is detectable in the fetus, and when mutation detection rates and disease allele frequencies vary among different ethnic groups. A classic example is the risk assessment for a fetus with echogenic bowel and only one detectable mutation in the cystic fibrosis transmembrane conductance regulator (CFTR) gene. The fetus could be affected or be a carrier on the basis of the presence of one detectable mutation. Accurate risk assessment in this scenario may be critical for parental decision making.

Cystic fibrosis is the most common severe autosomal recessive disorder; it affects about one in 2500 live births and has a carrier frequency of about one in 25 among European Caucasians. ${ }^{4}$ Cystic fibrosis is caused by homozygous mutations in the CFTR gene (OMIM 602421; 219700 cystic fibrosis; CFTR mutation database (http://www.genet.sickkids.on.ca/ (ftr/)). More than 1000 different CFTR mutations have been reported, and the mutation frequencies vary in different ethnic groups. The ultrasound finding of echogenic bowel, which is present in $0.1-0.2 \%$ of all pregnancies, may result from fetal meconium ileus and can result from cystic fibrosis. $^{56}$ The American College of Medical Genetics (ACMG) uses a panel of 25 CFTR mutations and advocates carrier screening for people with a family history of cystic fibrosis and their partners, as well as for all couples in the general population in whom the woman is pregnant or considering pregnancy. ${ }^{7}$ The clinical scenarios addressed in this paper are likely to be encountered with a high frequency. Despite the importance of accurate risk assessments in these scenarios, generalisable approaches to such risk assessments are lacking.

To facilitate accurate risk assessments, we developed novel, generalisable Bayesian methods to calculate the fetal risk of autosomal recessive disease when two independent risk factors are present and only one or no disease causing mutation is detected. Our approach is applicable to a wide variety of scenarios, including that of a fetus with echogenic bowel and one or no detectable CFTR mutation. We illustrate our approaches by using cystic fibrosis as a paradigm, but our methods can be applied to any autosomal recessive disorder, prenatally or postnatally, when only one or no mutation is identified with or without another risk factor.

\section{METHODS AND RESULTS}

Frequencies of echogenic bowel among fetuses who are affected by cystic fibrosis or carriers or noncarriers of cystic fibrosis

The frequencies of echogenic bowel among fetuses with and without cystic fibrosis were estimated as 14/126 (about 0.11)

\section{Key points}

- Risk assessment is an essential component of genetic counselling and testing, and Bayesian analysis plays a central role in complex risk calculations. Prenatal risk assessment for autosomal recessive diseases can be particularly complex when, for example, only one or no mutation is detectable in the fetus and when mutation detection rates and disease allele frequencies vary among different ethnic groups. A classic example is the risk assessment for a fetus with echogenic bowel and one detectable CFTR mutation.

- Novel, generalisable Bayesian methods were developed to calculate the autosomal recessive disease risk of a fetus when one or no mutation is detected and another independent risk factor is present. The methods can incorporate information such as genetic test results on either or both parents, each parent's ethnicity, and mutation detection rates for particular ethnicities. From the literature, the frequencies of echogenic bowel in a fetus affected with cystic fibrosis, an unaffected carrier, and an unaffected non-carrier for use in risk calculations were estimated as $0.11,0.00089$, and 0.00035 , respectively. For example, if a fetus with echogenic bowel has only one detectable CFTR mutation that is also present in their mother but no mutation is detected in a non-Hispanic Caucasian father who does not have a family history of cystic fibrosis, the posterior probability that the fetus is affected with cystic fibrosis is about 0.20 .

- The Bayesian method is versatile and generalisable, and it allows the probability of autosomal recessive disease to be calculated accurately, taking into account all relevant information.

and 128/346 428 (about 0.00037), respectively, by using data from a single large study. ${ }^{8}$ Of fetuses with echogenic bowel, only 11 had one detectable CFTR mutation after testing by an extended mutation detection panel that detects more than $99 \%$ of mutations in Brittany. ${ }^{8}$ If a frequency of cystic fibrosis carriers of one in 28 was assumed in Brittany, ${ }^{8}$ the frequencies of echogenic bowel among fetus carriers of cystic fibrosis and non-carriers would be calculated as 11/12 377 (about 0.00089) and 117/334 051 (about 0.00035), respectively. A statistically significant difference is seen between the frequencies of echogenic bowel among fetus carriers of cystic fibrosis and non-carriers $\left(p<0.005\right.$ by $\chi^{2}$ test). These frequencies can be used as conditional probabilities in the subsequent Bayesian analyses (see below). A number of 
Table 1 Summary of genetic risks for autosomal recessive disease in a fetus in various clinical settings*

\begin{tabular}{|c|c|c|c|}
\hline Fetus & Parent A & Parent B & Genetic risk for fetus \\
\hline One mutation identified & Not tested & Not tested & Scenario 1, table 4 \\
\hline One mutation identified & Tested negative & Not tested & Scenario 2 , table 5 \\
\hline One mutation identified & Same mutation identified & Not tested & Scenario 3 , table 6 \\
\hline One mutation identified & Same mutation identified & Tested, negative & Scenario 4 , table 7 \\
\hline One mutation identified & Same mutation identified & $\begin{array}{l}\text { Same or another mutation } \\
\text { identified }\end{array}$ & Disease risk negligible \\
\hline Tested negative & Not tested & Not tested & Scenario 5 , table 8 \\
\hline Tested negative & Tested negative & Not tested & Scenario 6 , table 9 \\
\hline Tested negative & Tested negative & Tested negative & Scenario 7 , table 10 \\
\hline Tested negative & One mutation identified & Not tested & $\begin{array}{l}\text { Scenario } 8 \text {, table } 11 \\
\text { (disease risk } \\
\text { negligible) }\end{array}$ \\
\hline Tested negative & One mutation identified & Tested negative & $\begin{array}{l}\text { Scenario 9, table } 12 \\
\text { (disease risk } \\
\text { negligible) }\end{array}$ \\
\hline Tested negative & One mutation identified & $\begin{array}{l}\text { Same or another mutation } \\
\text { identified }\end{array}$ & $\begin{array}{l}\text { Carrier and disease } \\
\text { risks negligible }\end{array}$ \\
\hline
\end{tabular}

*Genetic testing is assumed to be able to detect the same set of mutations in the fetus or parents, or both, and rates of de novo mutations and the risk of mosaicism are assumed to be negligible.

studies have estimated the frequency of cystic fibrosis among fetuses with echogenic bowel. ${ }^{9-15}$ The sizes of background populations were not determined, however, so to estimate accurately the frequency of echogenic bowel among fetus carriers of cystic fibrosis and non-carriers from these studies is impossible. One study estimated the frequency of echogenic bowel in the general population as 0.0023; however, the frequency of echogenic bowel among fetuses with cystic fibrosis was not determined. ${ }^{6}$ Another study determined the frequency of echogenic bowel among all fetuses with cystic fibrosis as about 0.55; however, echogenic bowel among fetuses without cystic fibrosis was not determined. ${ }^{16}$ The criteria for echogenic bowel are somewhat subjective and vary among centres, and it is important that the same criteria for echogenic bowel are used for fetuses with cystic fibrosis and fetus carriers and non-carriers, so we used data from the single large study by Scotet et al. for our calculations. $^{8}$

\section{Generalised Bayesian analyses for genetic risk calculations}

Table l summarises various situations encountered in clinical settings. The symbols used in this article are defined in table 2. Genetic risk assessments with tables 4-12 are discussed in detail below. The frequency of mutant alleles detectable by genetic testing among all mutant (disease) alleles is referred to as the "mutation detection rate" or "detection rate." We designated the probability that the fetus is positive for one risk factor (in this case, echogenic bowel) if the fetus is affected by cystic fibrosis, a carrier, or a noncarrier, as $a, b$, or $b^{\prime}$, respectively. We designated the detection rate of genetic testing performed on the fetus for the ethnic backgrounds of parent $\mathrm{A}$ and parent $\mathrm{B}$ as $c$ and $d$, respectively. For example, for a non-Hispanic Caucasian person, the sensitivity of genetic testing for the ACMG CFTR 25 mutation panel is 0.9. We designate the frequency of the mutation detected in the fetus among all disease alleles in the ethnic backgrounds of parent $\mathrm{A}$ and parent $\mathrm{B}$ as $e$ and $f$, respectively. For example, the frequency of $\triangle \mathrm{F} 508$ among all CFTR disease alleles in non-Hispanic Caucasian people is 0.7.

Table 3 summarises carrier frequencies of cystic fibrosis, overall mutation detection rates by the ACMG CFTR 25 mutation panel, and frequencies of major mutations for each major ethnic group for use in risk calculations. Tables 4-12 show our generalised Bayesian methods for calculating the risk of autosomal recessive disease in a variety of typical scenarios. Excel spreadsheets to facilitate risk calculations with these tables are available on request. The posterior probability for any particular column in our Bayesian analysis tables is the joint probability of that column divided by the sum of all joint probabilities in the table. To save space, we do not show posterior probabilities. In all examples, we assume that the de novo mutation rate and the risk of mosaicism are negligible. If risk factor number 1 (echogenic bowel in these examples) is not present, $a, b$, and $b^{\prime}$ can be replaced with 1 for risk calculations. We assume that the two different risk factors ("echogenic bowel" and "one detectable CFTR mutation") are independent; that is, one risk factor does not alter the probability of the other risk factor. If this were not the case, additional information would be needed for accurate risk assessment: for example, the conditional probability that the fetus has only one detectable mutation given echogenic bowel and affected status (or, alternatively, carrier status or non-carrier status). Sufficient data are lacking currently, however, to calculate such probabilities accurately.

Table 2 Definitions of symbols used in Bayesian analysis

\begin{tabular}{|c|c|}
\hline Symbol & Definition \\
\hline$p$ & Normal allele frequency \\
\hline$q$ & Disease allele frequency \\
\hline$a$ & $\begin{array}{l}\text { Probability that fetus is positive for risk factor number } 1 \text { if fetus } \\
\text { is affected }\end{array}$ \\
\hline$b$ & $\begin{array}{l}\text { Probability that the fetus is positive for risk factor number } 1 \text { if } \\
\text { fetus is a carrier }\end{array}$ \\
\hline$b^{\prime}$ & $\begin{array}{l}\text { Probability that the fetus is positive for risk factor number } 1 \text { if } \\
\text { fetus is a non-carrier }\end{array}$ \\
\hline c & $\begin{array}{l}\text { Mutation detection rate of genetic test for the ethnic } \\
\text { background of parent } A\end{array}$ \\
\hline$d$ & $\begin{array}{l}\text { Mutation detection rate of genetic test for the ethnic } \\
\text { background of parent } B\end{array}$ \\
\hline e & $\begin{array}{l}\text { Frequency of the mutation detected in the fetus among all } \\
\text { disease alleles in the ethnic background population of parent } A\end{array}$ \\
\hline$f$ & $\begin{array}{l}\text { Frequency of the mutation detected in the fetus among all } \\
\text { disease alleles in the ethnic background population of parent } B\end{array}$ \\
\hline$x$ & Prior carrier probability for parent $\mathrm{A}$ \\
\hline & Prior carrier probability for parent $B$ \\
\hline $\mathrm{Da}$ & Disease allele derived from parent $A$ \\
\hline $\mathrm{Db}$ & Disease allele derived from parent $B$ \\
\hline $\mathrm{N}$ & Normal allele \\
\hline
\end{tabular}


Table 3 Summary of carrier frequencies for cystic fibrosis, overall mutation detection rates by the ACMG 25 mutation panel, and frequencies of major mutations for each major ethnic group (adapted from Richards et al. and Bobadilla et al.) ${ }^{4} 18$

\begin{tabular}{|c|c|c|c|c|c|}
\hline \multirow{3}{*}{$\begin{array}{l}\text { Ethnic group } \\
\text { Non-Hispanic } \\
\text { Caucasian }\end{array}$} & \multirow{3}{*}{ 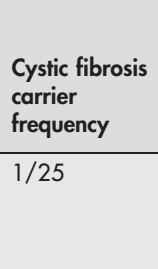 } & \multirow{3}{*}{$\begin{array}{l}\text { Overall mutation } \\
\text { detection rate by } \\
\text { ACMG CFTR } 25 \\
\text { mutation panel (\%) } \\
90\end{array}$} & \multirow{3}{*}{$\begin{array}{l}\text { Frequency } \\
\Delta 508 \text { among } \\
\text { all disease } \\
\text { alleles (\%) } \\
70\end{array}$} & & \\
\hline & & & & \multicolumn{2}{|c|}{ Other major mutations (\%)' } \\
\hline & & & & $\begin{array}{l}\text { G542X } \\
\text { G551D } \\
\text { W1282X } \\
\text { N1303K }\end{array}$ & $\begin{array}{l}2.4 \\
2.1 \\
1.4 \\
1.3\end{array}$ \\
\hline Ashkenazi Jewish & $1 / 25$ & 97 & 30 & $\begin{array}{l}\text { W1282X } \\
\text { G542X } \\
3849+10 \mathrm{kbC} \rightarrow \mathrm{T} \\
\mathrm{N} 1303 \mathrm{~K} \\
1717-1 \mathrm{G} \rightarrow \mathrm{A}\end{array}$ & $\begin{array}{l}48 \\
9.0 \\
6.0 \\
3.0 \\
1.0\end{array}$ \\
\hline African-American & $1 / 65$ & 69 & 48 & $\begin{array}{l}3120+1 \mathrm{G} \rightarrow \mathrm{A} \\
2307 \text { ins A } \\
\text { A559T } \\
\text { R553X } \\
\Delta \mathrm{F} 311 \\
\mathrm{G} 480 \mathrm{C} \\
405+3 \mathrm{~A} \rightarrow \mathrm{C} \\
\mathrm{S} 1255 \mathrm{X}\end{array}$ & $\begin{array}{l}12 \\
2.0 \\
2.0 \\
2.0 \\
2.0 \\
1.4 \\
1.4 \\
1.4\end{array}$ \\
\hline Hispanic American & $1 / 46$ & 57 & 46 & $\begin{array}{l}\text { G542X } \\
3849+10 \mathrm{kbC} \rightarrow T \\
\text { R1162X } \\
\text { R334W }\end{array}$ & $\begin{array}{l}5.4 \\
2.3 \\
1.6 \\
1.6\end{array}$ \\
\hline Asian American & $1 / 90$ & $?$ & 30 & $?$ & \\
\hline
\end{tabular}

*Mutation frequencies in non-Hispanic Caucasians based on data from a large, total American population collected by the Cystic Fibrosis Foundation. For each specific geographical region in Europe or frequencies of minor mutations, see Bobadilla et al. and Lao et al. ${ }^{18}$

Scenarios 1-4 illustrate the situation of one detectable mutation in a fetus with echogenic bowel. Scenarios 5-9 illustrate the situation of no detectable mutation in a fetus with echogenic bowel.

\section{Scenario 1: fetus with one detectable mutation, neither parent tested}

In scenario 1, neither parent is tested. Even though the ACMG appropriately advocates carrier screening of parents before prenatal testing, this scenario is, unfortunately, not uncommon-in part, because echogenic bowel usually occurs late in pregnancies, when setting up carrier testing before prenatal testing can be problematic. In scenario l, the issue of non-paternity or non-maternity can be ignored. In addition, as a formal introduction to our methods, it makes the most sense to start with examples in which less genetic information is available and then describe how the accuracy of risk assessments increases as more information is obtained. Table 4 shows an example where, in addition to echogenic bowel, another risk factor is present; that is, one mutation is detected by genetic testing of the fetus. If the fetus is affected, the probability that Da (the disease allele derived from parent $\mathrm{A}$; see table 2) is identified as a particular mutation is $e$ and the probability that $\mathrm{Db}$ is undetectable is $1-d$. The joint probability for column A thus is calculated as 0.25 axye $(1-d)$. The joint probabilities of the other columns in table 4 are calculated similarly. The posterior probability that the fetus is affected is the sum of the joint probabilities of columns A and B divided by the sum of all joint probabilities. The generalised approach shown in table 4 allows calculation of the risk of cystic fibrosis for a fetus of mixed descent (northern European or other ethnic backgrounds, or both) or the risk of other autosomal recessive diseases for which frequency distributions of disease alleles, mutation detection rates, and the frequency of other risk factors are known. If a relative of parent $\mathrm{A}$ or parent $\mathrm{B}$ is affected or an obligate carrier, this table can still be applied when neither that relative nor any other family member has been tested.

An example of the use of table 4 is if a fetus has echogenic bowel and only one detectable mutation $(\Delta \mathrm{F} 508)$, neither parent has a family history of cystic fibrosis, and neither parent has been tested. If $x=0.04, y=0.04, a=0.11$, $b=0.00089, \quad b^{\prime}=0.00035, c=0.97, d=0.9, e=0.3$, and $f=0.7$ (for example, when the mother (parent A) is Ashkenazi Jewish and the father (parent B) is nonHispanic Caucasian, see table 3 ), the posterior probability that the fetus is affected is about 0.11 . If the prior disease risk for the fetus is higher, because of a higher carrier risk for either parent, then the posterior probability that the fetus is affected is higher. For example, if $x=0.04, y=0.5, a=0.11$, $b=0.00089, b^{\prime}=0.00035, c=0.97, d=0.9, e=0.3$, and $f=0.7$ (for example, when the mother (parent A) is Ashkenazi Jewish and the father (parent B) is non-Hispanic Caucasian, see table 3 ), then the posterior probability that the fetus is affected is about 0.15 .

Scenario 2: fetus with one detectable mutation, one parent tests negative, other parent not tested

Table 5 shows the Bayesian analysis for scenario 2, in which parent A tests negative and parent $\mathrm{B}$ has not been tested. As no mutation is found in parent $\mathrm{A}$, the conditional probability that Da is detected or undetected is 0 or 1 , respectively. The posterior probability that the fetus is affected is the joint probability of column A divided by the sum of all joint probabilities. Note that the posterior carrier probabilities of parent $\mathrm{A}$ and parent $\mathrm{B}$ also are calculated simultaneously in table 5. For example, the posterior carrier probability of parent $\mathrm{B}$ is the sum of the joint probabilities in columns A, B, C, D, G, and $\mathrm{H}$ divided by the sum of all of the joint probabilities. If a relative of parent $\mathrm{A}$ or parent $\mathrm{B}$ is affected or an obligate carrier, this table can still be applied when neither that relative nor any other family member has been tested. 
Table 4 Generalised Bayesian analysis for autosomal recessive disease with two independent risk factors (scenario 1)*

\begin{tabular}{|c|c|c|c|c|c|c|c|}
\hline Variable & Generalised Baye & esian analysis vo & ariables & & & & \\
\hline $\begin{array}{l}\text { Prior probabilities } \\
\text { Parent A }\end{array}$ & & & & & & & \\
\hline Carrier & & & $x$ & & & & \\
\hline Non-carrier & & & & & & $1-x$ & \\
\hline Parent B & & & & & & & \\
\hline Carrier & & y & & & & y & \\
\hline Non-carrier & & & & & $1-y$ & & $1-y$ \\
\hline Conditional probabilities & & & & & & & \\
\hline Fetus & & & & & & & \\
\hline Affected $(D a+D b)$ & 0.25 & & & & & & \\
\hline Carrier (Da) & & & 0.25 & & 0.5 & & \\
\hline Carrier (Db) & & & & 0.25 & & 0.5 & \\
\hline Non-carrier & & & & & & & 1 \\
\hline Risk factor 1 (that is, echogenic bowel) & $a$ & & $b$ & $b$ & $b$ & $b$ & $b^{\prime}$ \\
\hline One mutation identified & & & & & & & \\
\hline $\mathrm{Da}$ & e & & $e$ & & e & & \\
\hline $\mathrm{Db}$ & & $f$ & & $f$ & & $f$ & \\
\hline- & & & & & & & 0 \\
\hline No other mutation detected & & & & & & & \\
\hline $\mathrm{Db}$ & $1-d$ & & & & & & \\
\hline $\mathrm{Da}$ & & $1-c$ & & & & & \\
\hline $\mathrm{N}$ & & & 1 & 1 & 1 & 1 & 1 \\
\hline Joint probability & 0.25 axye $(1-d)$ & $0.25 \operatorname{axyf}(1-c)$ & 0.25 bxye & 0.25 bxyf & $0.5 b x(1-y)$ e & 0.5 by $(1-x) f$ & 0 \\
\hline Column & A & B & C & $\mathrm{D}$ & $\mathrm{E}$ & $\mathrm{F}$ & G \\
\hline
\end{tabular}

For example, if $x=0.04, y=0.04, a=0.11, b=0.00089$, $b^{\prime}=0.00035, c=0.97$, and $d=0.9$ (for example, when the mother (parent A) is Ashkenazi Jewish and the father (parent B) is non-Hispanic Caucasian), the posterior probability that the fetus is affected is about 0.072 , which is lower than in the example in scenario l. If $x=0.04, y=1 /$ $46, a=0.11, b=0.00089, b^{\prime}=0.00035, c=0.9$, and $d=0.57$ (for example, when the mother (parent A) is non-Hispanic Caucasian and the father (parent B) is Hispanic), the posterior probability that the fetus is affected is about 0.20 .

\section{Scenario 3: fetus with one detectable mutation} present in one parent, the other parent not tested Table 6 shows an example in which the fetus has been found to have one detectable mutation and parent $\mathrm{A}$ has the same mutation, but no genetic testing has been performed on parent B. In this case, the carrier probability of parent B is designated $y$. As parent $\mathrm{A}$ is a carrier, only four possible scenarios exist: carrier parent B and affected fetus, carrier parent $\mathrm{B}$ and carrier fetus with a mutation derived from parent A, carrier parent B and carrier fetus with a mutation from parent $\mathrm{B}$, and non-carrier parent $\mathrm{B}$ and carrier fetus. If the fetus is affected, Db must be undetectable, as Da is the one mutation that is detected. If the fetus is affected, the conditional probability that $\mathrm{Db}$ is undetectable is $1-d$. If the fetus is a carrier with a disease allele from parent B $(\mathrm{Db})$, then the mutation in the fetus is the same as the mutation in parent A only by chance. The probability that a mutation inherited by the fetus from parent $\mathrm{B}$ is the same as the mutation carried by parent $\mathrm{A}$ is simply the frequency of that mutation among all disease alleles in the ethnic background population of parent $\mathrm{B}$, or $f$. The posterior probability that the fetus is affected is the joint probability in column A divided by the sum of all joint probabilities. If a relative of parent B is affected or an obligate carrier, this table can still be applied when neither that relative nor any other family member has been tested.

An example of the use of table 6 is if a fetus has echogenic bowel and one detectable mutation $(\Delta \mathrm{F} 508)$, parent $\mathrm{A}$ has the same mutation present in the fetus, parent $\mathrm{B}$ has no family history of cystic fibrosis, and parent $\mathrm{B}$ has not been tested. If $y=0.04, a=0.11, b=0.00089, d=0.9$, and $f=0.7$ (for example, when the father (parent B) is non-Hispanic Caucasian), the posterior probability that the fetus is affected is about 0.20 .

\section{Scenario 4: fetus with one detectable mutation} present in one parent, the other parent tests negative Table 7 shows an example in which parent A has the same mutation that is detected in the fetus and parent $\mathrm{B}$ has no identifiable mutation by genetic testing. Although the situation seems similar to that in table 6, differences exist. If the fetus is affected, we now know that the mutation from parent B is undetectable, with a probability of 1 (column A). If the fetus is a carrier with a disease allele $\mathrm{Db}$, the probability that $\mathrm{Db}$ is detectable is 0 (column $\mathrm{C}$ ). The probability that the fetus is affected is the joint probability in column A divided by the sum of all joint probabilities. Note that the posterior probability that the fetus is affected in table 7 is very similar to that in table 6 . This is because the joint probabilities of columns B and C are very small compared with those of columns A and D, which are essentially the same in tables 6 and 7. If a relative of parent $B$ is affected or an obligate carrier, this table can still be applied when neither that relative nor any other family member has been tested.

An example of the use of table 7 is if a fetus has the same mutation detected in the mother (parent A), the mutation detected is $\Delta \mathrm{F} 508$, the father (parent $\mathrm{B}$ ) has no family history of cystic fibrosis, and the father tests negative. If $y=0.04$, $a=0.11, b=0.00089, d=0.9$, and $f=0.7$ (for example, when the father (parent B) is non-Hispanic Caucasian), the posterior disease risk of the fetus is about 0.20 .

\section{Scenario 5: fetus with no detectable mutation, neither parent tested}

Scenario 5 (table 8) shows an example in which the fetus does not have any identifiable mutation and neither parent has been tested. If the fetus is affected, the probability that both paternal and maternal mutations are undetectable is $(1-c)(1-d)$. The posterior probability that the fetus is affected is the joint probability in column A divided by the sum of all joint probabilities. If a relative of parent $\mathrm{A}$ or parent $\mathrm{B}$ is affected or an obligate carrier, this table can still be applied 
Table 5 Generalised Bayesian analysis for autosomal recessive disease with two independent risk factors (scenario 2)*

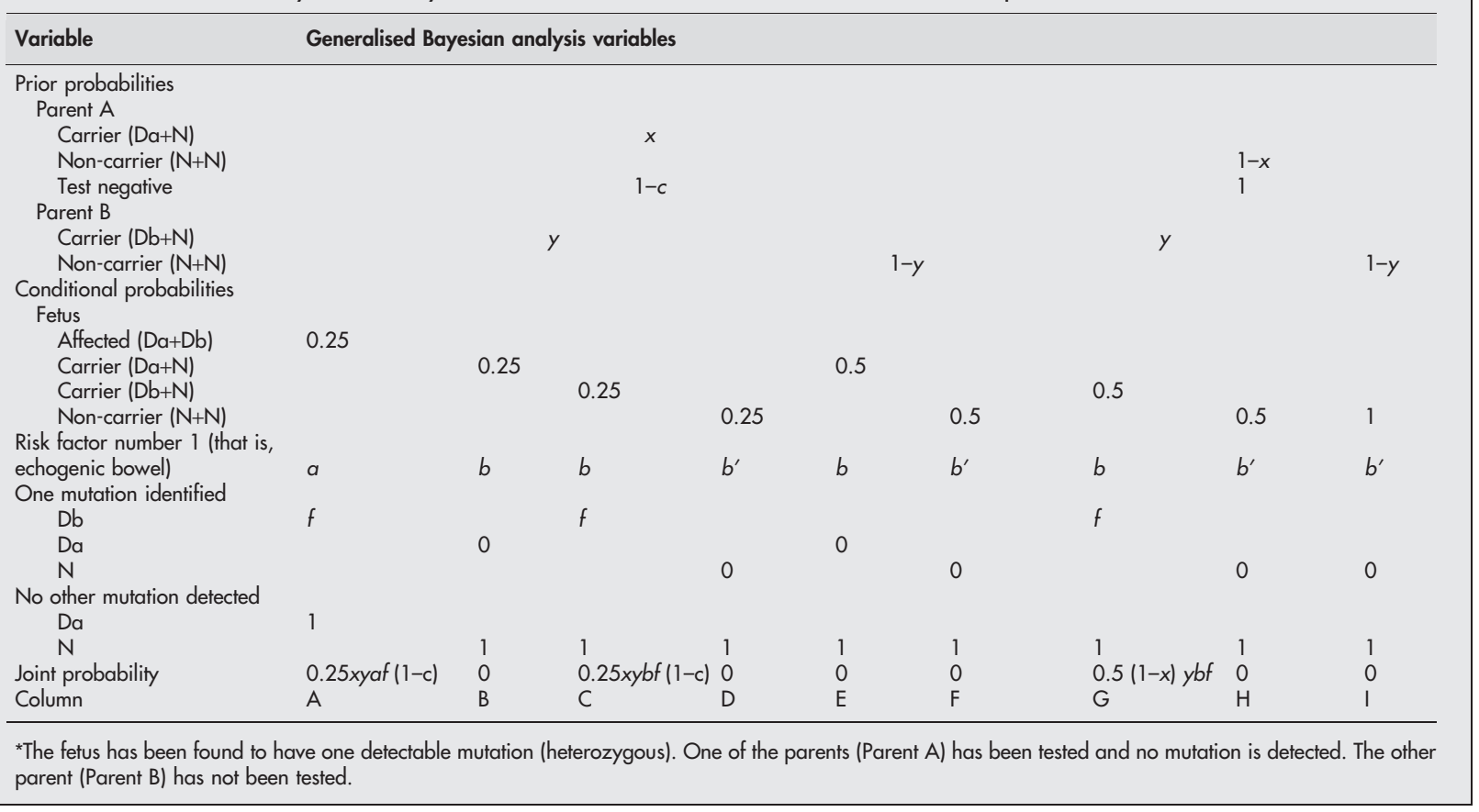

when neither that relative nor any other family member has been tested.

An example of the use of table 8 is if a fetus has echogenic bowel but does not have any detectable mutation, neither parent has been tested, and neither parent has a family history of cystic fibrosis. If $x=0.04, y=0.04, a=0.11$, $b=0.00089, \quad b^{\prime}=0.00035, c=0.97, d=0.9, e=0.3$, and $f=0.7$ (for example, when the mother (parent A) is Ashkenazi Jewish and the father (parent B) is nonHispanic Caucasian), the posterior probability that the fetus is affected or a carrier is 0.00039 or 0.0067 , respectively. If the prior disease risk for the fetus is higher because of a higher carrier risk for either parent, then the posterior probability that the fetus is affected is higher. For example, if $x=0.04, y=0.5, a=0.11, b=0.00089, b^{\prime}=0.00035, c=0.97$, $d=0.9, e=0.3$, and $f=0.7$ (for example, when the mother (parent A) is Ashkenazi Jewish and the father (parent B is
non-Hispanic Caucasian), then the posterior probability that the fetus is affected or a carrier is about 0.0059 or 0.079 , respectively.

Scenario 6: fetus and one parent with no detectable mutation, and the other parent not tested

Table 9 shows an example in which the fetus does not have any identifiable mutation, no mutation is detected in parent A, and parent B has not been tested. If the fetus is affected, the probability that both paternal and maternal mutations are undetectable is $(1-d)$, because parent A's mutation, if present, would not be detected. The posterior probability that the fetus is affected is the joint probability in column A divided by the sum of all joint probabilities. If a relative of parent A or parent B is affected or an obligate carrier, this table can still be applied when neither that relative nor any other family member has been tested.

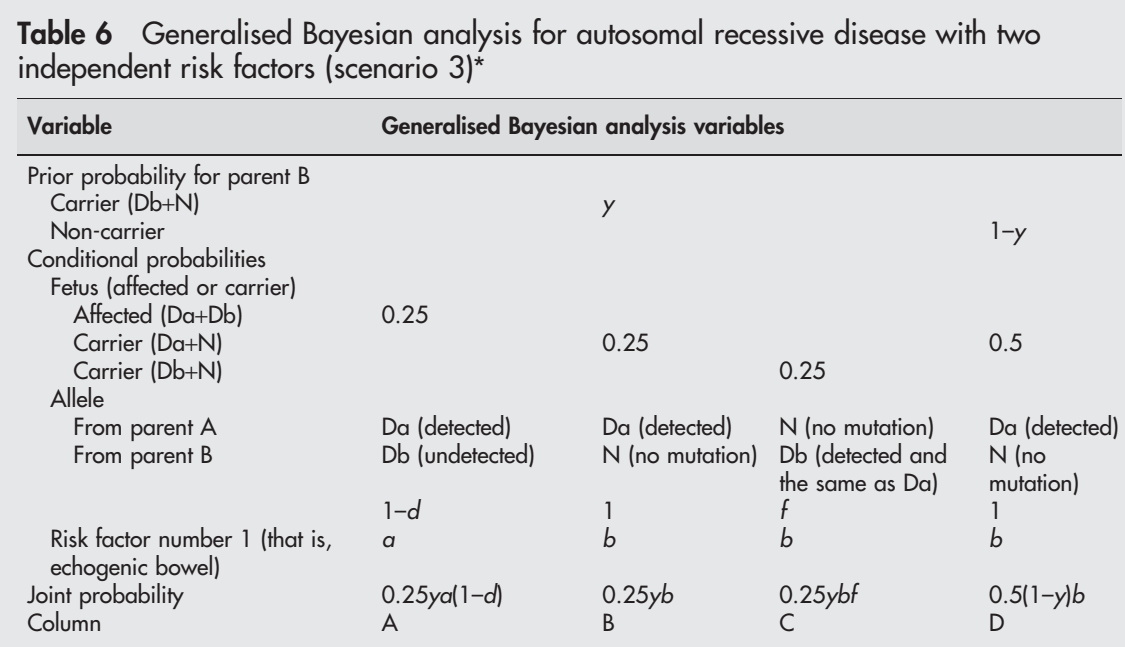

*Fetus found to have one detectable mutation (heterozygous). One of the parents (parent A) has the mutation detected in the fetus, but the other parent (parent B) not tested. 


\begin{tabular}{|c|c|c|c|c|}
\hline \multirow{2}{*}{ 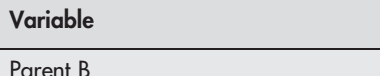 } & \multicolumn{4}{|c|}{ Generalised Bayesian analysis variables } \\
\hline & & Carrier $(\mathrm{Db}+\mathrm{N})$ & & Non-carrier \\
\hline Prior probability & & & & $1-y$ \\
\hline $\begin{array}{l}\text { Conditional probability of negative } \\
\text { genetic test }\end{array}$ & & $1-d$ & & 1 \\
\hline \multicolumn{5}{|l|}{ Conditional probabilities } \\
\hline \multicolumn{5}{|l|}{ Fetus (affected or carrier) } \\
\hline Affected $(D a+D b)$ & 0.25 & & & \\
\hline Carrier $(\mathrm{Da}+\mathrm{N})$ & & 0.25 & & 0.5 \\
\hline Carrier $(\mathrm{Db}+\mathrm{N})$ & & & 0.25 & \\
\hline \multicolumn{5}{|l|}{ Alleles } \\
\hline \multirow{3}{*}{$\begin{array}{l}\text { From parent } A \\
\text { From parent } B\end{array}$} & Da (detected) & Da (detected) & $N$ (no mutation) & Da (detected) \\
\hline & $\mathrm{Db}$ (undetected) & $\mathrm{N}$ (no mutation) & $\mathrm{Db}$ (detected) & $\mathrm{N}$ (no mutation) \\
\hline & 1 & 1 & 0 & 1 \\
\hline $\begin{array}{l}\text { Risk factor number } 1 \\
\text { (echogenic bowel) }\end{array}$ & $a$ & $b$ & $b$ & $b$ \\
\hline Joint probability & $0.25 y a(1-d)$ & $0.25 y b(1-d)$ & 0 & $0.5(1-y) b$ \\
\hline Column & & B & C & \\
\hline
\end{tabular}

An example of the use of table 9 is if a fetus has echogenic bowel but does not have any detectable mutation, one parent (parent A) tested negative, the other parent (parent B) has not been tested, and neither parent has a family history of cystic fibrosis. If $x=0.04, y=0.04, a=0.11, b=0.00089$, $b^{\prime}=0.00035, c=0.97, d=0.9, e=0.3$, and $f=0.7 \quad$ (for example, when the mother (parent A) is Ashkenazi Jewish and the father (parent B) is non-Hispanic Caucasian), the posterior probability that the fetus is affected or a carrier is 0.00040 or 0.0068 , respectively.

\section{Scenario 7: fetus and both parents with no detectable mutation}

Table 10 shows an example where the fetus does not have any identifiable mutation and no mutation is detected in parent A or parent B. If the fetus is affected, the probability that both paternal and maternal mutations are undetectable is 1 , because both of the parents' mutations, if present, would be undetectable. The posterior probability that the fetus is affected is the joint probability in column A divided by the sum of all joint probabilities. If a relative of parent A or parent $\mathrm{B}$ is affected or an obligate carrier, this table can still be applied when neither that relative nor any other family member has been tested.

An example of the use of table 10 is if a fetus has echogenic bowel but does not have any detectable mutation, both parents test negative, and neither parent has a family history of cystic fibrosis. If $x=1 / 65, y=1 / 46, a=0.11, b=0.00089$, $b^{\prime}=0.00035, c=0.69$, and $d=0.57$ (for example, when the mother (parent A) is African-American and the father (parent B) is a Hispanic), the posterior probability that the fetus is affected or a carrier is 0.0035 or 0.018 , respectively.

Scenario 8: fetus with no detectable mutation, one parent with a detectable mutation, other parent not tested

Table 11 shows an example in which parent A has a detectable mutation, no mutation is detected in the fetus, and parent B has not been tested. In this scenario, the risk of the fetus being affected is negligible; that is, the posterior probability of column A is essentially 0 . If a relative of parent $\mathrm{B}$ is affected or an obligate carrier, this table can still be applied when neither that relative nor any other family member has been tested.

Table 8 Generalised Bayesian analysis for autosomal recessive disease with one risk factor and no identifiable mutation (scenario 5)*

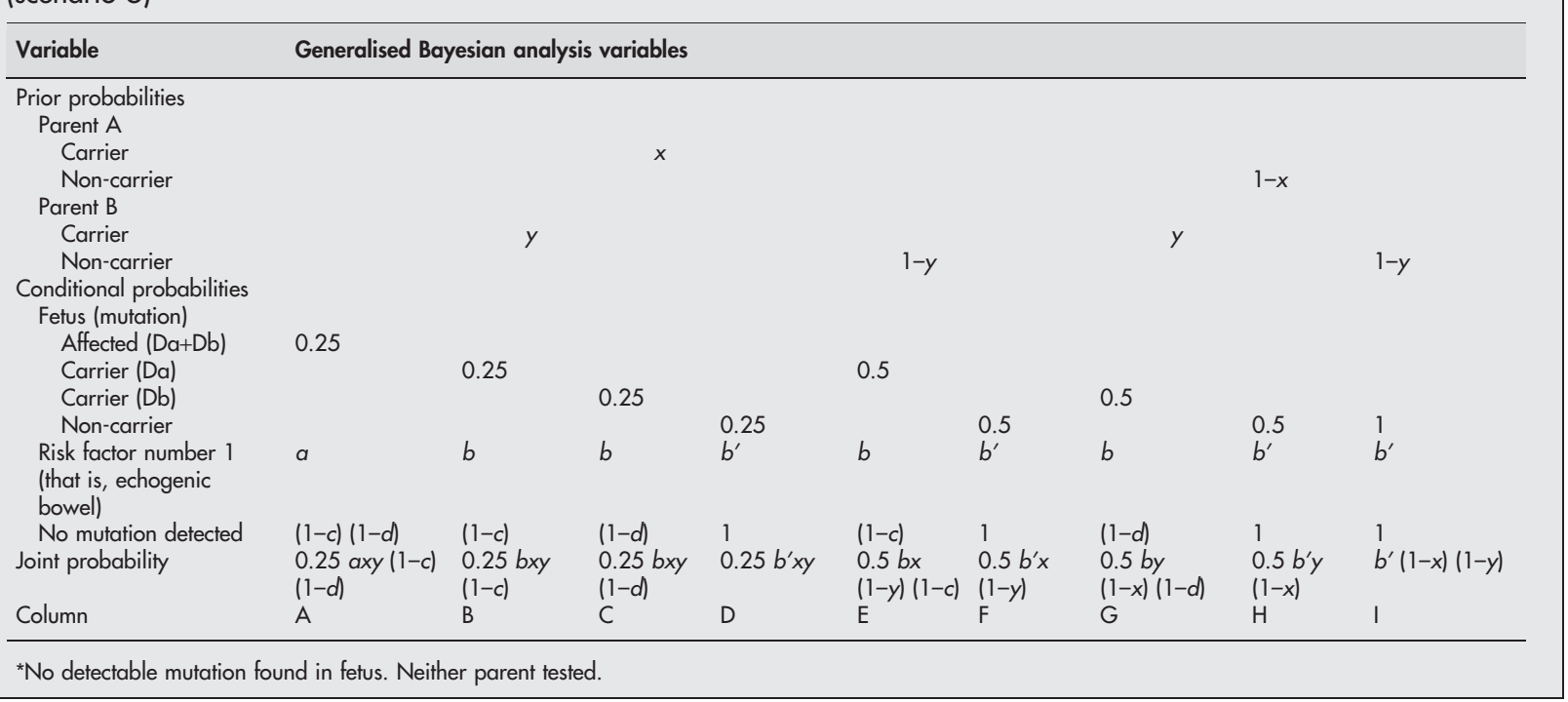


Table 9 Generalised Bayesian analysis for autosomal recessive disease with one risk factor and no identifiable mutation (scenario 6)*

\begin{tabular}{|c|c|c|c|c|c|c|c|c|c|}
\hline Variable & Generalised & ayesian anc & ysis variable: & & & & & & \\
\hline Prior probabilities & & & & & & & & & \\
\hline Parent A & & & & & & & & & \\
\hline Carrier & & & $x$ & & & & & & \\
\hline Non-carrier & & & & & & & & $1-x$ & \\
\hline Conditional probability & & & & & & & & & \\
\hline Test negative & & & $1-c$ & & & & & 1 & \\
\hline Parent B & & & & & & & & & \\
\hline Carrier & & $y$ & & & & & $y$ & & \\
\hline Non-carrier & & & & & $1-y$ & & & & $1-y$ \\
\hline $\begin{array}{l}\text { Conditional probabilities } \\
\text { Fetus (mutation) }\end{array}$ & & & & & & & & & \\
\hline $\begin{array}{l}\text { refus (mutafion) } \\
\text { Affected (Da+Db) }\end{array}$ & 0.25 & & & & & & & & \\
\hline Carrier (Da) & & 0.25 & & & 0.5 & & & & \\
\hline Carrier (Db) & & & 0.25 & & & & 0.5 & & \\
\hline Non-carrier & & & & 0.25 & & 0.5 & & 0.5 & 1 \\
\hline Risk factor number 1 & $a$ & $b$ & $b$ & $b^{\prime}$ & $b$ & $b^{\prime}$ & $b$ & $b^{\prime}$ & $b^{\prime}$ \\
\hline No mutation detected & $(1-d)$ & 1 & $(1-d)$ & 1 & 1 & 1 & $(1-d)$ & 1 & 1 \\
\hline Joint probability & $\begin{array}{l}0.25 \text { axy } \\
(1-c)(1-d)\end{array}$ & $\begin{array}{l}0.25 b x y \\
(1-c)\end{array}$ & $\begin{array}{l}0.25 \text { bxy } \\
(1-c)(1-d)\end{array}$ & $\begin{array}{l}0.25 b^{\prime} x y \\
(1-c)\end{array}$ & $\begin{array}{l}0.5 b x(1-y) \\
(1-c)\end{array}$ & $\begin{array}{l}0.5 b^{\prime} x \\
(1-y)(1-c)\end{array}$ & $\begin{array}{l}0.5 \text { by } \\
(1-x)(1-d)\end{array}$ & $\begin{array}{l}0.5 b^{\prime} y \\
(1-x)\end{array}$ & $b^{\prime}(1-x)(1-y)$ \\
\hline Column & A & B & C & D & $\mathrm{E}$ & $\mathrm{F}$ & $G$ & $\mathrm{H}$ & I \\
\hline
\end{tabular}

*No detectable mutation has been found either in the fetus, or in one of the parents (parent A). The other parent (parent B) has not been tested. Note the differences from Table 8.

An example of the use of table 11 is if a fetus has echogenic bowel but does not have any detectable mutation, one parent (parent A) tests positive for a mutation, and the other parent (parent B), who does not have a family history of cystic fibrosis, has not been tested. If $y=1 / 65, a=0.11, b=0.00089$, $b^{\prime}=0.00035, c=0.9$, and $d=0.69$ (for example, when the mother (parent A) is non-Hispanic Caucasian, and the father (parent B) is African-American), the posterior probability that the fetus is affected or a carrier is 0 or 0.0061 , respectively.

Scenario 9: fetus and one parent with no detectable mutation, and the other parent with a mutation identified

Table 12 shows an example in which parent $\mathrm{A}$ has a detectable mutation but no mutation is detected in the fetus or parent B. In this scenario, the risk of the fetus being affected is negligible; that is, the posterior probability of column A is essentially 0 . If a relative of parent B is affected or an obligate carrier, this table can still be applied when neither that relative nor any other family member has been tested.

An example of the use of table 12 is if a fetus has echogenic bowel but does not have any detectable mutation, one parent (parent B) does not have a family history of cystic fibrosis, tested negative, and the other parent (parent A) has a detectable mutation. If $y=1 / 46, a=0.11, b=0.00089$, $b^{\prime}=0.00035, c=0.9$, and $d=0.57$ (for example, when the mother (parent A) is non-Hispanic Caucasian, and the father (parent B) is Hispanic), the posterior probability that the fetus is affected or a carrier is 0 or 0.012 , respectively.

\section{DISCUSSION}

Bayesian analysis plays an essential role in calculations of carrier or disease risks for autosomal recessive disorders. ${ }^{1-3}$ Generalised methods to calculate disease risks when only one

Table 10 Generalised Bayesian analysis for autosomal recessive disease with one risk factor and no identifiable mutation (scenario 7)*

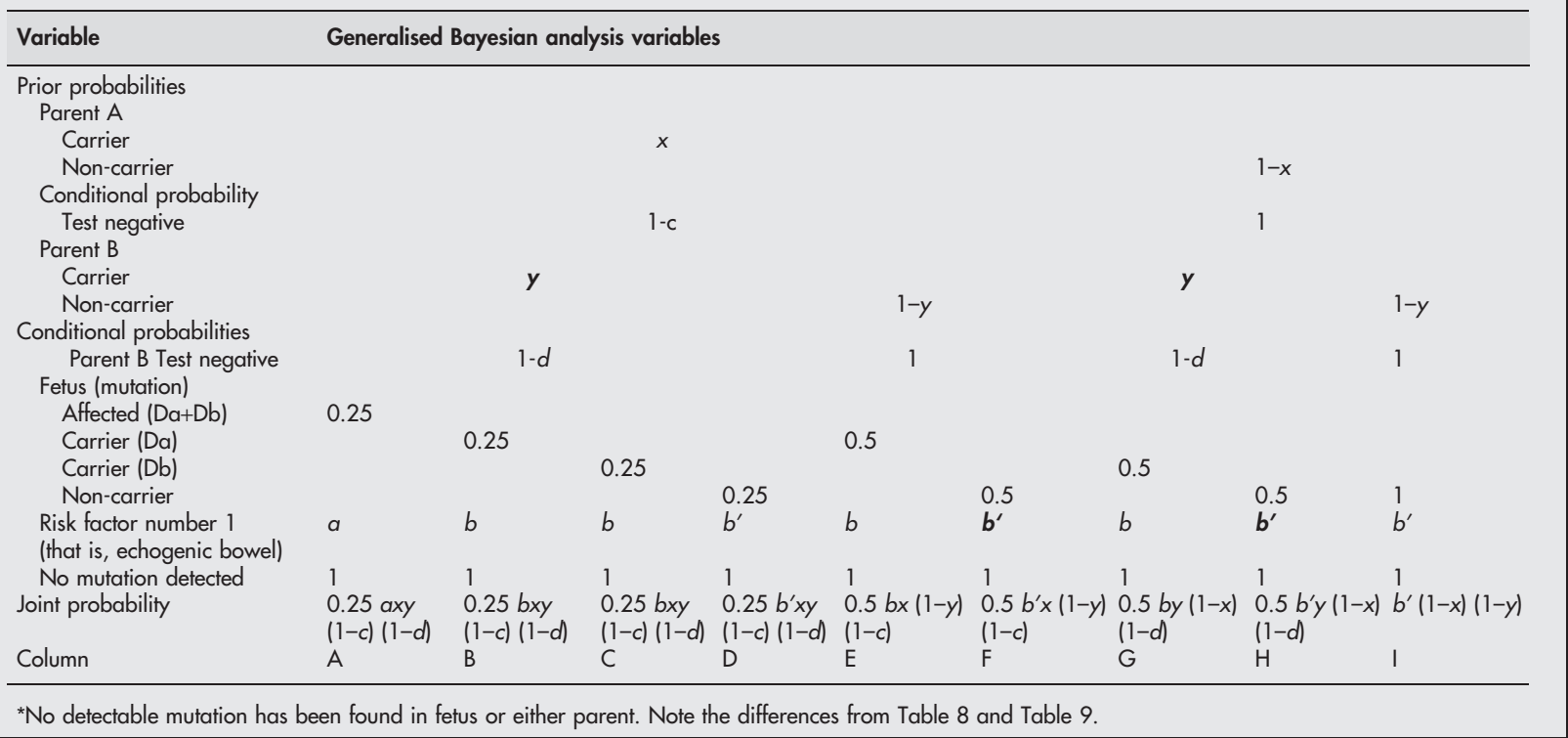


Table 11 Generalised Bayesian analysis for autosomal recessive disease with one risk factor and no identifiable mutation (scenario 8)*

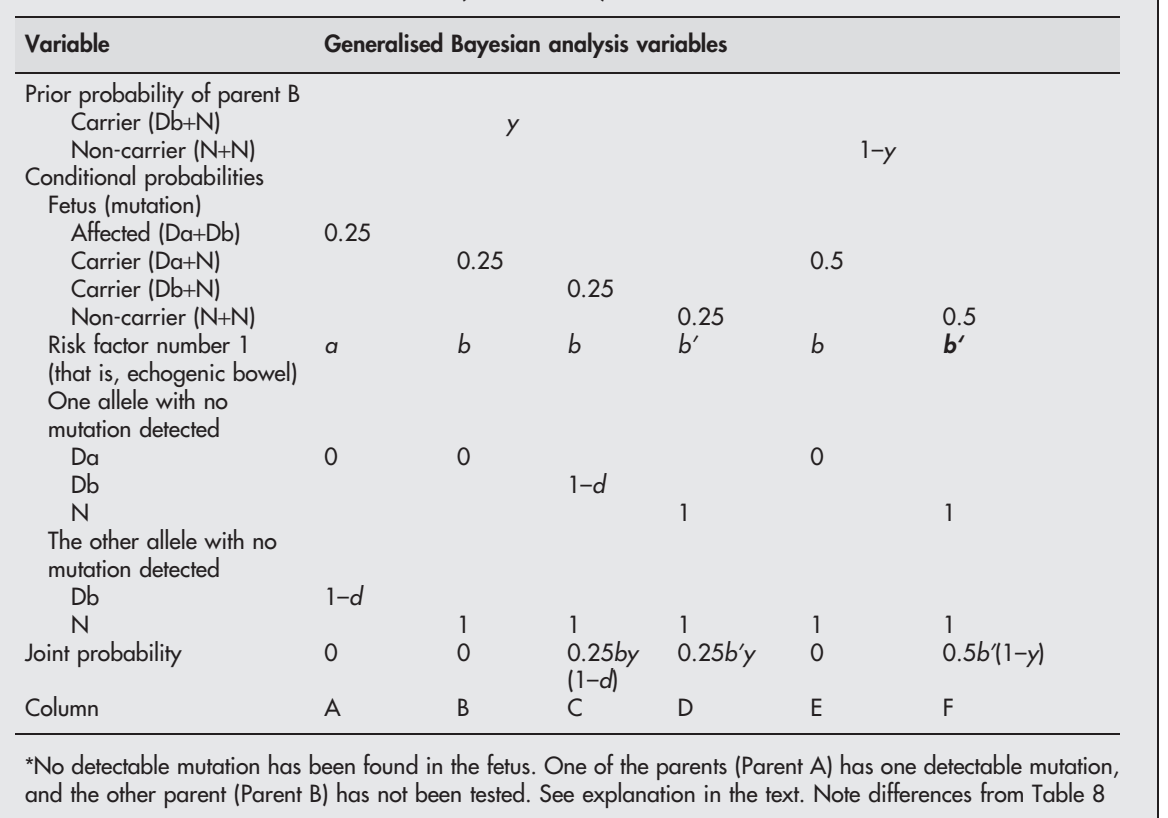

or no mutation is detected in an autosomal recessive disease for which multiple mutations have been identified in the disease gene are lacking. We have described generalisable Bayesian methods to calculate risks for any autosomal recessive disease. Excel spreadsheets to facilitate the risk calculations are available upon request. Our comprehensive set of Bayesian analysis methods allows calculations of the risks of cystic fibrosis for fetuses of parents with different ethnic backgrounds in a variety of clinical settings; that is, disease risks can be calculated even when the mutation detection rate and the frequency of particular disease alleles vary among different ethnic populations. Our methods are particularly useful for cystic fibrosis, but they are generalisable to other autosomal recessive diseases. Our methods also can be applied to postnatal scenarios, as long as the conditional probabilities of a particular risk factor are known.

Although echogenic bowel usually is detected rather late in pregnancy, proper counselling and testing should include both parents. We have shown how the accuracy of risk assessments increases as more information becomes available (see tables 1 and 4-12): risk assessment is most accurate when both parents, in addition to the fetus, are tested. Genetic counselling for couples with a fetus with echogenic bowel is beyond the capability of the average obstetrician; all such couples should be referred to a medical geneticist or genetic counsellor, or both.

Hodge et al. described risk calculations for a fetus with echogenic bowel and one characterised CFTR mutation,

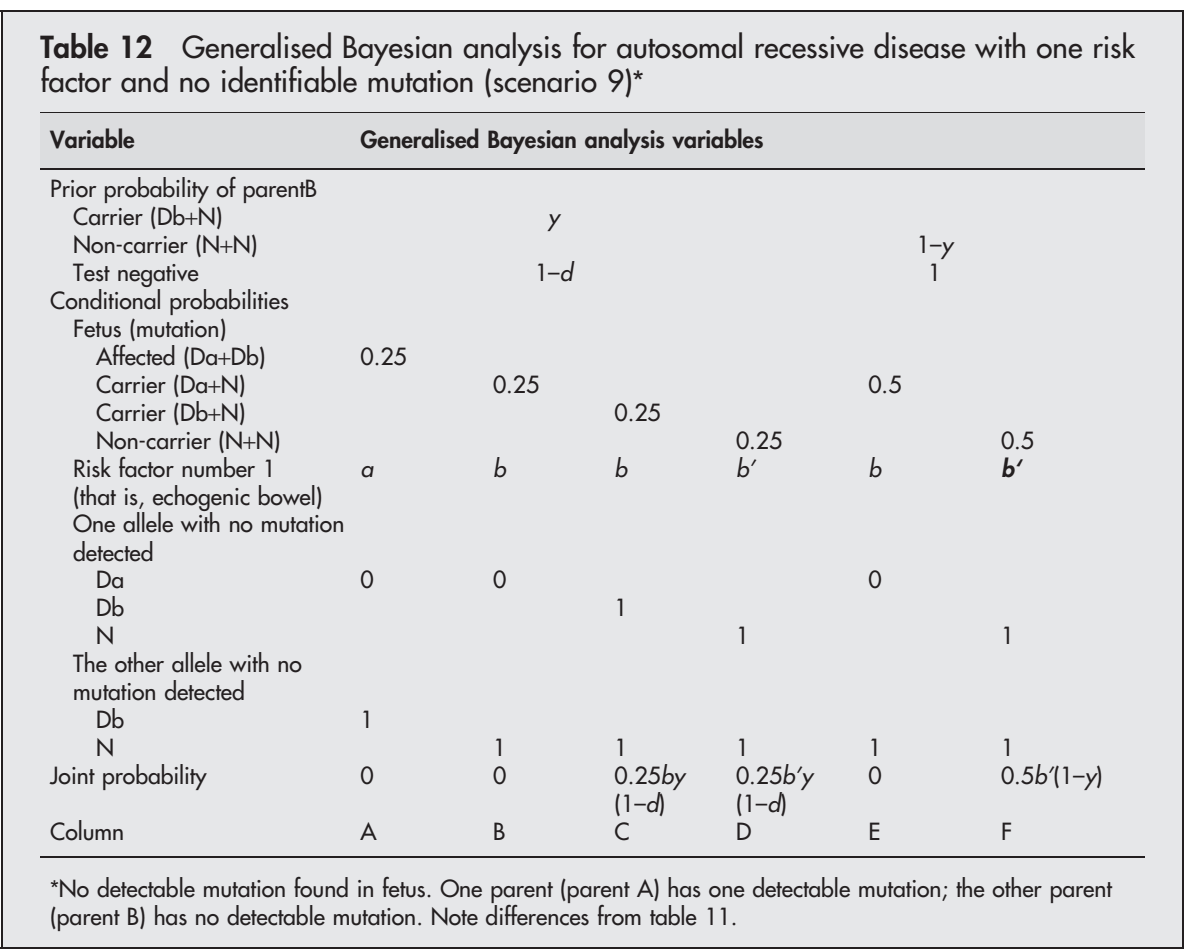


$\Delta$ F508. ${ }^{17}$ The authors carefully and thoroughly evaluated relevant information and provided useful illustrations to explain the relevant Bayesian analyses. Their methods did not take into account, however, the variable frequency of $\triangle$ F508 alleles among all CFTR disease alleles in parents of various ethnicities, which is relevant to the example in table 1 of their report. This table would be useful for a situation in which the fetus tests positive, but the identity of the mutation is unknown. As the fetus in the actual example was known to have $\Delta \mathrm{F} 508$, however, the frequency of $\Delta \mathrm{F} 508$ should be used, rather than the overall mutation detection rate, for one of the parents. The authors also assumed that the frequency of echogenic bowel and whether a fetus was a carrier or non-carrier were independent; however, this assumption later was shown by Scotet et al. to be invalid, ${ }^{8}$ as our $b$ and $b^{\prime}$ are different. Another important factor is the conditional probability of echogenic bowel if the fetus is affected, a carrier, or a non-carrier. Hodge et al., instead of using conditional probabilities in their risk calculations, used "risk due to mutation analysis" and "risk due to ultrasound, ${ }^{\prime \prime 17}$ the latter of which is the prevalence of an affected fetus among all fetuses with echogenic bowel-that is, the positive predictive value of ultrasound in their setting. The positive predictive value (usually obtained by Bayesian analysis) is influenced by disease prevalence (that is, the prior probabilities) and therefore may be subject to ascertainment bias. Generalisation of positive predictive values therefore may be problematic.

Our calculations assume that the frequency of echogenic bowel is the same for cystic fibrosis with different CFTR mutations; that is, echogenic bowel and one identified CFTR mutation are independent risk factors. This may not always be true: cystic fibrosis with severe mutations, such as $\Delta \mathrm{F} 508$, may be associated with an increased probability of echogenic bowel. Hodge et al., however, suggested that only a slight increase was seen in the risk of disease in the fetus if echogenic bowel and one identified CFTR mutation were not assumed to be conditionally independent relative to the assumption of conditional independence. ${ }^{17}$

\section{Conclusion}

Our Bayesian methods are versatile and generalisable, and they allow the probability of autosomal recessive disease to be calculated accurately, taking into account all relevant information, including genetic test results on either or both the parents, the ethnic background of each parent, the overall mutation detection rate for each parent's ethnicity, and the frequency of a mutation, if detected, among all disease alleles for a particular ethnicity.

\section{ACKNOWLEDGEMENTS}

We thank Hanna Rennert for critical reading of the manuscript and insightful discussions.

\section{NOTES ADDED IN PROOFS}

1. The ACMG CFTR mutation panel will soon be reduced to 23 mutations; however, that will not significantly alter any of our calculations.

2. Conditional probabilities of echogenic bowel should be updated when the data on ethnicity specific frequencies of echogenic bowel for each condition (cystic fibrosis disease, cystic fibrosis carrier or non-carrier) are available.

3. Our most recent review on Bayesian risk calculations visually illustrates the principles of Bayesian analysis. ${ }^{20}$

\section{Authors' affiliations}

S Ogino, Department of Pathology, Brigham and Women's Hospital, Boston, Massachusetts, USA

S Ogino, Department of Medical Oncology, Dana-Farber Cancer Institute, Boston, Massachusetts, USA

S Ogino, Harvard Medical School, Boston, Massachusetts, USA R B Wilson, Department of Pathology and Laboratory Medicine, University of Pennsylvania Medical Center, Philadelphia, Philadelphia, USA

W W Grody, Departments of Pathology and Laboratory Medicine, Human Genetics, and Pediatrics, UCLA School of Medicine, Los Angeles, California, USA

Conflicts of interest: None declared.

Correspondence to: Dr Ogino, Department of Pathology, Brigham and Women's Hospital, Harvard Medical School, 75 Francis Street, Boston, MA 02115 USA; sogino@partners.org

\section{REFERENCES}

1 Bridge PJ. The calculation of genetic risks: worked examples in DNA diagnostics. Baltimore: Johns Hopkins University Press, 1997.

2 Young ID. Introduction to risk calculation in genetic counselling. Oxford: Oxford University Press, 1999.

3 Ogino S, Wilson RB. Genetic testing and risk assessment for spinal muscular atrophy (SMA). Hum Genet 2002; 11 1:477-500.

4 Richards CS, Bradley LA, Amos J, Allitto B, Grody WW, Maddalena A, McGinnis MJ, Prior TW, Popovich BW, Watson MS, Palomaki GE. Standards and guidelines for CFTR mutation testing. Genet Med 2002;4:379-91.

5 Irish MS, Ragi JM, Karamanoukian H, Borowitz DS, Schmidt D, Glick PL. Prenatal diagnosis of the fetus with cystic fibrosis and meconium ileus. Pediatr Surg Int 1997; 12:434-6.

6 Dicke JM, Crane JP. Sonographically detected hyperechoic fetal bowel: significance and implications for pregnancy management. Obstet Gynecol 1992;80:778-82.

7 Grody WW, Cutting GR, Klinger KW, Richards CS, Watson MS, Desnick RJ. Laboratory standards and guidelines for population-based cystic fibrosis carrier screening. Genet Med 2001;3:149-54.

8 Scotet V, De Braekeleer M, Audrezet MP, Quere I, Mercier B, Dugueperoux I, Andrieux J, Blayau M, Ferec C. Prenatal detection of cystic fibrosis by ultrasonography: a retrospective study of more than 346000 pregnancies. J Med Genet 2002;39:443-8.

9 Al-Kouatly HB, Chasen ST, Streltzoff J, Chervenak FA. The clinical significance of fetal echogenic bowel. Am J Obstet Gynecol 2001;185:1035-8.

10 Monaghan KG, Feldman GL. The risk of cystic fibrosis with prenatally detected echogenic bowel in an ethnically and racially diverse North American population. Prenat Diagn 1999;19:604-9.

11 MacGregor SN, Tamura R, Sabbagha R, Brenhofer JK, Kambich MP, Pergament E. Isolated hyperechoic fetal bowel: significance and implications for management. Am J Obstet Gynecol 1995;173:1254-8.

12 Ferriman EL, Mason G, Ellis L. Risk of cystic fibrosis with prenatally detected echogenic bowel. Prenat Diagn 1999;19:1177.

13 Muller F, Dommergues M, Simon-Bouy B, Ferec C, Oury JF, Aubry MC, Bessis R, Vuillard E, Denamur E, Bienvenu T, Serre JL. Cystic fibrosis screening: a fetus with hyperechogenic bowel may be the index case. J Med Genet 1998;35:657-60.

14 Sepulveda W, Leung KY, Robertson ME, Kay E, Mayall ES, Fisk NM. Prevalence of cystic fibrosis mutations in pregnancies with fetal echogenic bowel. Obstet Gynecol 1996;87:103-6.

15 Slotnick RN, Abuhamad AZ. Prognostic implications of fetal echogenic bowel. Lancet 1996;347:85-7.

16 Boue A, Muller F, Nezelof C, Oury JF, Duchatel F, Dumez Y, Aubry MC, Bove J. Prenatal diagnosis in 200 pregnancies with a 1-in-4 risk of cystic fibrosis. Hum Genet 1986;74:288-97.

17 Hodge SE, Lebo RV, Yesley AR, Cheney SM, Angle H, Milunsky J. Calculating posterior cystic fibrosis risk with echogenic bowel and one characterized cystic fibrosis mutation: avoiding piffalls in the risk calculations. Am J Med Genet 1999;82:329-35.

18 Bobadilla JL, Macek M Jr, Fine JP, Farrell PM. Cystic fibrosis: a worldwide analysis of CFTR mutations--correlation with incidence data and application to screening, Hum Mutat 2002; 19:575-606

19 Lao O, Andres AM, Mateu E, Bertranpetit J, Calafell F. Spatial patterns of cystic fibrosis mutation spectra in European populations. Eur J Hum Genet 2003;11:385-94.

20 Ogine S, Wilson RB. Bayesian analysis and risk assessment in genetic counselling and testing. J Mol Diagn 2004;6:1-9. 